Z. Klin. Chem. Klin. Biochem.

13. Jg. 1975 , S. $437-444$

\title{
Über alkalische Phosphatasen in menschlichen Faeces, Dünndarmschleimhaut und Galle und das Vorkommen von 5' $^{\prime}$-Nucleotidase in Faeces
}

\author{
Von Vera Benic und Marijana Fišer-Herman \\ Aus der Klinik für Infektionskrankheiten (Vorstand: Prof. Dr. J. Fališevac) der Medizinischen Fakultät der \\ Universität Zagreb
}

(Eingegangen am 30. Oktober 1974/13. Mai 1975)

Zusammenfassung: Die alkalische Phosphatase (EC 3.1.3.1) aus normalen Faeces gleicht der alkalischen Phosphatase in Extrakten aus Dünndarmschleimhaut, von welcher sie sich nur durch etwas größere Beweglichkeit in Stärkegelelektropherogrammen unterscheidet. Zwischen den Eigenschaften der alkalischen Phosphatase in Extrakten aus normalen und acholischen Faeces wurden keine Unterschiede gefunden. Alkalische Phosphatase aus Blasengalle scheint in Faeces nicht unverändert vorzukommen. In den Faeces von Patienten mit akuter Virushepatitis oder Protozoasis kommt eine alkalische Phosphatase vor, welche Eigenschaften einer $5^{\prime}$-Nucleotidase (EC 3.1.3.5) hat und aus der Galle zu stammen scheint. In Stühlen, welche dieses Enzym enthalten ist die Gesamtaktivität der alkalischen Phosphatase viel geringer als in normalem oder acholischem Stuhl.

\section{Alkaline phosphatases in human feces, intestinal mucosa and bile, and the occurrence of $5^{\prime}$-nucleotidase in feces}

Summary: Alkaline phosphatase (EC 3.1.3.1) in extracts of human feces resembles alkaline phosphatase in extracts of duodenal mucosa, except for its electrophoretic mobility in starch gel. It is very probable that the normal feces alkaline phosphatase derives from intestinal mucosa. Gall bladder alkaline phosphatase, which is markedly different, has not been found in normal feces. Some patients with acute viral hepatitis or protozoasis excrete an alkaline phosphatase which resembles gall bladder alkaline phosphatase and has the characteristics of $5^{\prime}$-nucleotidase (EC 3.1.3.5). The appearance of this enzyme correlates with low total alkaline phosphatase activity of the excreta.

\section{Einführung}

Es ist seit langem bekannt, daß menschliche Faeces viel alkalische Phosphatase (EC 3.1.3.1) enthalten. Wir versuchten die Eigenschaften der in Faeces vorkommenden -alkalischen Phosphatase kennen zu lernen und womöglich zu ermitteln, woher sie stammt. Deshalb verglichen wir alkalische Phosphatase aus Faeces mit der aus Duodenalsaft, Blasengalle und Dünndarmschleimhaut. Úber einen Teil dieser Versuche haben wir früher berichtet (1).

Wir berichten jetzt über Ergebnisse von weiteren Untersuchungen der alkalischen Phosphatase in Faeces von gesunden und kranken Personen, sowie in Extrakten aus Dünndarmschleimhaut und Blasengalle. Besonders beschäftigten wir uns mit einer auf Stärkegelelpherogrammen sichtbaren Fraktion der alkalischen Phosphatase, welche wir ursprünglich nur in Faeces von Protozoen- trägern beobachtet hatten und deren Eigenschaften sich von denen der übrigen alkalischen Phosphatase der Faeces unterscheiden.

\section{Material und Methoden}

Faeces

Frische Faeces wurden bei $4^{\circ} \mathrm{C}$ längstens 24 Stunden aufbewahrt und von jeder Portion ein Teil bei $100^{\circ} \mathrm{C}$ bis zur Gewichtskonstanz getrocknet. Untersucht wurden Faeces von folgenden Personen:

$\begin{array}{lr}\text { Gesunde Kinder, 2-8 Jahre alt } & 10 \\ \text { Kinder mit akuter Virushepatitis } & 45 \\ \text { Gesunde Erwachsene } & 15 \\ \text { Erwachsene mit Leberkrankheiten: } & \\ \text { a) obstruktiver Gelbsucht } & 23 \\ \text { b) Lebercirrhose } & 13 \\ \text { c) akuter Virushepatitis } & 4 \\ \text { Erwachsene mit Lambliasis } & 40 \\ & \text { Insgesamt } 150\end{array}$ 
Je $2 \mathrm{~g}$ der homogenisierten Faeces wurden mit der 5 fachen Menge physiologischer NaCl-Lösung verrieben und bei $2500 \mathrm{U} /$ min 15 Minuten zentrifugiert. Sichtbar fetthaltige Extrakte wurden mit $2 / 3$ ihres Volumens an $n$-Butanol gemischt, 24 Stunden bei $4^{\circ} \mathrm{C}$ gehalten, zentrifugiert und die Butanolschicht verworfen. Die wäßrigen Extrakte wurden sofort verarbeitet oder bei $-18^{\circ} \mathrm{C}$ aufbewahrt.

\section{Blasengalle}

Galle stammte aus den Gallenblasen von 10 frischen Leichen an deren Leber, Gallengängen und Darmtrakt makroskopisch keine Veränderungen festgestellt worden waren. $50 \mathrm{ml}$ eines Gemisches von annähernd gleichen Mengen Galle wurden mit $100 \mathrm{ml}$ physiologischer $\mathrm{NaCl}-\mathrm{Lösung}$ vermischt, 30 Minuten bei $2500 \mathrm{U} / \mathrm{min}$ zentrifugiert, der visköse Teil abgetrennt und die Lösung wie Faecesextrakt behandelt.

Darmschleim haut

Je $30 \mathrm{~cm}$ vom Jejunum derselben Leichen wurden sofort bei $-18^{\circ} \mathrm{C}$ eingefroren. Nach dem Auftauen wurde etwa $100 \mathrm{~g}$ Schleimhaut abgeschabt, mit der doppelten Wassermenge bei $4{ }^{\circ} \mathrm{C}$ homogenisiert und die Emulsion mit $2 / 3$ ihres Volumens an $n$-Butanol extrahiert. Der fast klare wäßrige Extrakt wurde teils sofort verarbeitet, teils bei $-18^{\circ} \mathrm{C}$ aufbewahrt.

Bestimmung der Phosphataseak tivität

Die Aktivität der alkalischen Phosphatase wurde nach Kirberger \& Martini (2) bei pH 9 und nach King \& Armstrong (3) bei $\mathrm{pH} 10$ bestimmt und in King-Armstrong-Einheiten ${ }^{1}$ ) pro $\mathrm{kg}$ trockener Faeces, bżw. 1 nativer Galle, bzw. kg frischer Dünndarmschleimhaut ausgedrückt.

\section{Inhibition durch $L$-Phenylalanin}

Der Aktivitätsabfall der Enzymlösungen in Gegenwart von $5 \mathrm{mmol} / \mathrm{l} L$-Phenylalanin wurde nach Fishman \& Ghosh bei pH 9 und pH 10 bestimmt (4).

\section{Denaturierung durch Harnstoff}

Die Restaktivität der Phosphataselösungen wurde nach Fenelly et al. (5) in Gegenwart von $2 \mathrm{~mol} / \mathrm{l}$ Harnstoff bei pH 9 und .pH 10 gemessen.

\section{Inaktivierung bei $56^{\circ} \mathrm{C}$}

Nach Vorschrift von Posen et al (6) wurde der Aktivitätsverlust der 15 Minuten auf $56^{\circ} \mathrm{C}$ erwärmten Enzymlösungen bei $\mathrm{pH} 9$ und $\mathrm{pH} 10$ gemessen.

Alle Aktivitätsverluste sind in Prozent der ursprünglichen Aktivität ausgedrückt.

\section{Elektrophoretische Trennung der Fraktionen}

Die Elektrophorese der alkalischen Phosphatase-Lösungen wurde auf horizontalen Stärkegelplatten nach Smithies (7) im diskontinuierlichen Puffersystem nach Poulik (8) ausgeführt, die Lage der Enzymfraktionen mit $\alpha$-Naphthylphosphat und Fast Blue RR nach Bamford (9) sichtbar gemacht und mit im gleichen Versuch aufgetrennten Serumeiweißfraktionen verglichen.

\section{Reinigung der Enzymlösungen durch Gelfiltration}

Die alkalische Phosphatase enthaltenden Extrakte wurden 30 Minuten mit $20000 \mathrm{U} / \mathrm{min}$ zentrifugiert und durch Kolonnen von Sephadex G 100 filtriert. Kolonnendurchmesser $4 \mathrm{~cm}$, Schich tlänge $100 \mathrm{~cm}$, Anquellen des Gels und Elution mit $0,1 \mathrm{~mol} / \mathrm{l}$ Carbonat-Bicarbonat-Puffer $\mathrm{pH} 9$, Durchlauf $18,6 \mathrm{ml}$ pro Stunde. Mit Hilfe eines automatischen Kollektors wurden je 70 Portionen von 6,2 ml gesammelt und ihre optische Dichte bei $280 \mathrm{~nm}$ registriert. Alle alkalische Phosphatase enthaltenden

1) Umrechnung in internationale Einheiten (U) erfolgte nicht, da eine Diskrepanz zwischen empirischem Umrechnungsfaktor (4,0; Südhof et al., Deut. Med. Wochenschr. 87, 249(1962) und theoretischem Wert $(7,1)$ besteht.
Fraktionen einer Kolonne wurden lyophilisiert und unmittelbar vor der Weiterverarbeitung in $10 \mathrm{ml}$ dest. Wasser gelöst.

Bestimmung der spezifischen Aktivität und $\mathrm{K}_{\mathrm{m}}$-Werte Die spezifischen Aktivitäten der durch Gelfiltration gereinigten alkalischen Phosphatasen wurden mit Phenylphosphat in Carbonat-Bicarbonat Puffer in Gegenwart von 2,0, 5,0, 6,25, 8,5, 12,5 und $25 \mathrm{mmol} / 1$ Substrat bei $\mathrm{pH} 8,0,8,5,9,0,9,5,10,0$, 10,5 und 11,0 gemessen und in $\dot{\mu} g$ Phenol pro $\mathrm{ml}$ Enzymlösung ausgedrückt.

$\mathrm{K}_{\mathrm{m}}$-Werte wurden nach Lineweaver \& Burk bestimmt.

Bestimmung der 5'-Nucleotidase-(EC 3.1.3.5)-Aktivität 5'-Nucleotidase-Aktivität wurde mit AMP nach Campbell (10) mit und ohne Zusatz von $0,1 \mathrm{~mol} / \mathrm{l}$ Nickelchlorid gemessen.

\section{Ergebnisse}

\section{Stärkegelelektrophorese der Rohextrakte}

Die Lage der stärksten alkalische Phosphatase enthaltenden Fraktionen in Stärkegelelektropherogrammen von nativen Extrakten aus Faeces, Blasengalle und Dünndarmschleimhaut, verglichen mit dem Serumproteinogramm und den Isoenzymen der alkalischen Phosphatasen im Serum zeigt Abbildung 1 unserer früheren Arbeit (1).

Auf Abbildung 1 sind alle beobachteten alkalische Phosphatase enthaltenden Fraktionen aus Serum, Faeces, Galle und Darmschleimhaut schematisch dargestellt.

Abbildung 2 zeigt die Häufigkeit der alkalische Phos: phatase enthaltenden Fraktionen in Faecesproben von Gesunden und Kranken.

Die Fraktionen 1 und 1 a kamen in Faeces von gesunden Kindern und Erwachsenen regelmäßig vor, während in Elektropherogrammen von Gallenextrakten an diesen Stellen nichts zu sehen war. Fraktion 3 haben wir in einem Fünftel der untersuchten Faecesproben und im

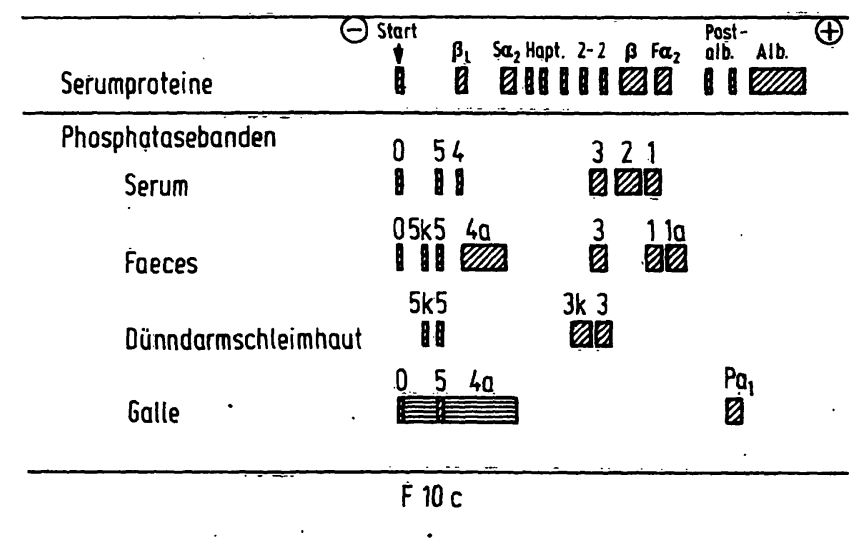

Abb. 1. Ửbersicht der auf Stärkegelelektropherogrammen beobachteten alkalischen Phosphatase-Fraktionen. Die Lage der Fraktion 1 entspricht der sogenannten Leberphosphatase des Serums; Fraktion 3 der sogenannten intestinalen Serümphosphatase; die Fraktionen 4 und 5 wurden im Serum von Kranken mit Stauungsikterus beobachtet; „a“ bedeutet anodenwärts verschoben, „,k“ verschoben zu Kathode, „O" Auftragstelle. 

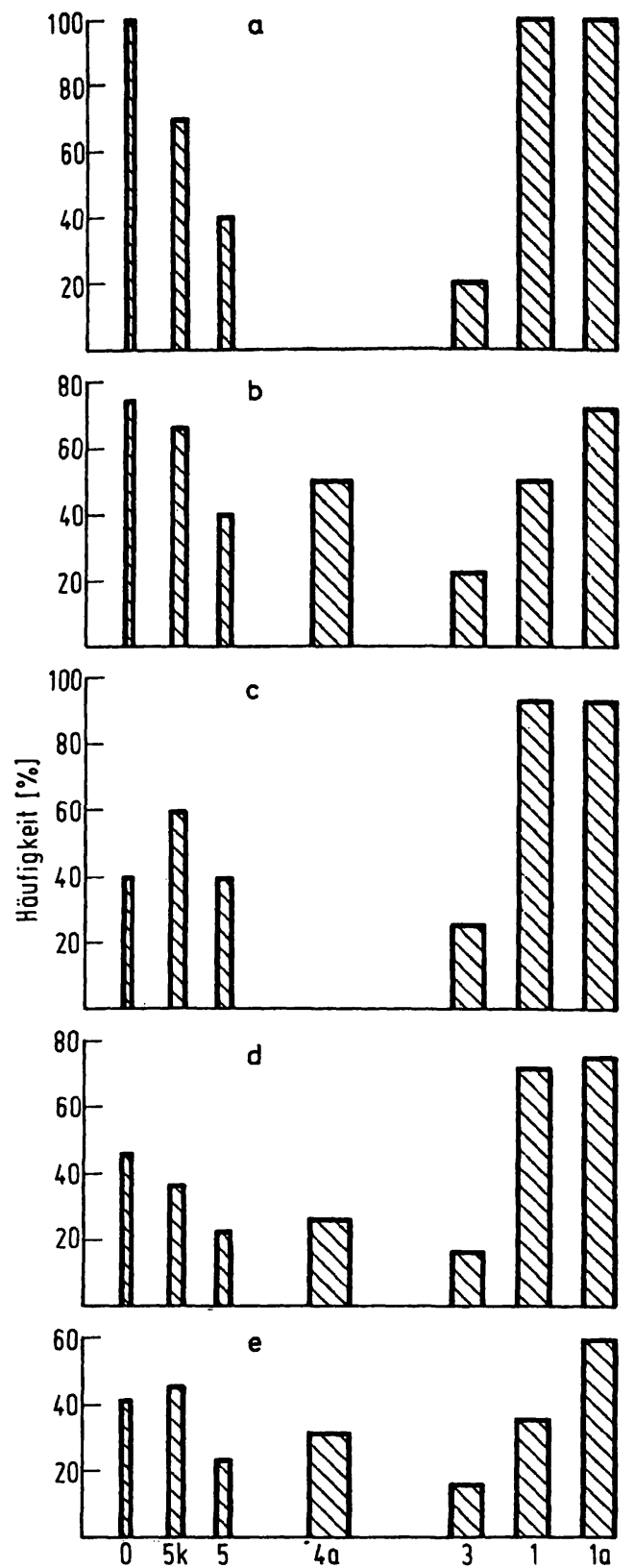

Abb. 2. Häufigkeit der auf Stärkegelelektropherogrammen beobachteten alkalischen Phosphatasenfraktionen in $\mathbf{1 5 0}$ Faecesproben von gesunden und kranken Personen: a) gesunde Kinder, 3-8 Jahre $(n=10)$ b) Kinder, 3-10 Jahre, akute Virushepatitis $(n=45)$ c) Gesunde Erwachsene ( $n=15)$ d) Erwachsene, Lebererkrankungen $(n=40)$ e) Erwachsene, Lambliasis $(n=40)$.

Extrakt aus Darmschleimhaut gefunden. Die Fraktion 4 a bildete stets einen breiten Streifen und kam nur bei Leberfunktionsstörungen oder Protozoasis vor. In Stühlen mit dieser Fraktion war die Gesamtaktivität der alkalische Phosphatase niedrig, die Fraktion 1 und 1 a waren schwach oder gar nicht sichtbar, häufig war nur 4a zu sehen. Tabelle 1 zeigt die Häufigkeit des Auftretens dieser Fraktion.
Tab. 1. Frequenz der Phosphatasenfraktion $4 \mathrm{a}$ in Stuhlextrakten

\begin{tabular}{|c|c|c|c|}
\hline & & \multicolumn{2}{|c|}{$\begin{array}{l}\text { Elektropherographisch } \\
\text { gefunden }\end{array}$} \\
\hline & $\begin{array}{l}\text { Zainl } \\
\text { der } \\
\text { Fälle }\end{array}$ & $\begin{array}{l}\text { Nur } \\
\text { Fraktion 4a }\end{array}$ & $\begin{array}{l}\text { Fraktion } 4 \text { a } \\
\text { und } 1 \\
\text { oder } 1 \text { a }\end{array}$ \\
\hline \multicolumn{4}{|l|}{ Gesunde Kinder } \\
\hline $\begin{array}{l}\text { 3-8 Jahre } \\
\text { Kinder mit akuter Virus- }\end{array}$ & 10 & 0 & 0 \\
\hline hepatitis, 3-10 Jahre & 45 & 9 & 14 \\
\hline $\begin{array}{l}\text { Gesunde Erwachsene } \\
\text { Erwachsene mit } \\
\text {. }\end{array}$ & 15 & 0 & 0 \\
\hline $\begin{array}{l}\text { Leberkrankheiten: } \\
\text { obstruktive Gelbsucht }\end{array}$ & 23 & 3 & 2 \\
\hline Lebercirrhose & 13 & 3 & 2 \\
\hline akute Virushcpatitis & 4 & 0 & 1 \\
\hline Erwachsene mit Lambliasis & 40 & 10 & 3 \\
\hline
\end{tabular}

Gesamtaktivität der alkalische Phosphatase in Stühlen von Gesunden und Kranken

In der Tabelle 2 sind die in Stühlen von 150 Personen gefundenen Aktivitäten in King-Armstrong-Einheiten ${ }^{1}$ ) pro kg Trockensubstanz angegeben. Merkwürdigerweise waren die durchschnittlichen Aktivitäten in Stühlen sowohl von gesunden und kranken Kindern als auch von Erwachsenen in welchen die Fraktion 4 a fehlte, kaum verschieden. Die Streuung der Befunde war aber sehr groß, und zwar bei Erwachsenen wesentlich größer als bei Kindern. Die Affinität der alkalischen Phosphatase aus Faeces für Phenylphosphat war bei $\mathrm{pH} 10$ viel größer als bei $\mathrm{pH} 9$.

Mit dem Auftrennen der Fraktion 4a änderte sich das Bild: die Aktivität der alkalischen Phosphatase war herabgesetzt und bei $\mathrm{pH} 10$ wurden niedrigere Werte gemessen als bei pH 9. In Stühlen die nur die Fraktion 4 a enthielten, wurden nur geringe Aktivitäten gefunden.

Inhibition der alkalischen Phosphatase in Faecesextrakten durch $L$-Phenylalanin, Inaktivierung bei $56{ }^{\circ} \mathrm{C}$ und Harnstoffdenaturierung

Unterschiede zwischen der Fraktion 4a und den übrigen in Faeces vorkommenden Fraktionen zeigten sich auch bei Inhibitions- und Inaktivierungsversuchen. Ein Teil dieser Resultate ist auf der Abb. 3 dargestellt.

Abbildung 3 zeigt, daß die Aktivität der alkalischen Phosphatase der Faeces ohne Fraktion 4 a durch $L$-Phenylalanin stark herabgesetzt wurde; bei $\mathrm{pH} 9$ um 70-80\%; bei pH 10 um $50-60 \%$.

In Extrakten, welche die Fraktion 4a enthielten, wurden die alkalische Phosphatase durch $L$-Phenylalanin weit weniger inhibiert, besonders bei $\mathrm{pH} 9$, bei welchem diese Fraktion gegenüber dem Inhibitor fast vollkommen resistent zu sein schien.

Extrakte ohne Fraktion 4a verloren in 15 Minuten bei $56^{\circ} \mathrm{C}$ etwa ein Fünftel ihrer Aktivität; bei pH 9 etwas mehr als bei pH 10. Die Fraktion 4a wurde bei pH 9 
Tab. 2. Mittelwerte und Standardabweichungen der alkalischen Phosphatasenaktivitäten der Faeces. King-Armstrong Einheiten pro kg Trockensubstanz

\begin{tabular}{|c|c|c|c|c|c|c|c|}
\hline & & & pH 9 & & $\mathrm{pH} 10$ & & $\overline{\mathrm{X}} \mathrm{pH} 10 /$ \\
\hline & & $\mathbf{N}$ & $\mathbf{X}$ & $\mathbf{s}$ & $\mathbf{X}$ & $\mathbf{s}$ & X pH 9 \\
\hline \multirow[t]{3}{*}{ I } & $\begin{array}{l}\text { Stühle in welchen die Fraktion } 4 \text { a } \\
\text { nicht gefunden worden war: }\end{array}$ & & & & & & \\
\hline & $\begin{array}{l}\text { - von gesunden Kindern } \\
\text { - von Kindern mit akuter Virus }\end{array}$ & 10 & 302300 & 150700 & 596200. & 269200 & 1,96 \\
\hline & $\begin{aligned} & \text { Hepatitis } \\
& \text { - von gesunden Erwachsenen } \\
& \text { - von Erwachsenen mit Leberkrankheiten } \\
& \text { - von Erwachsenen mit Lambliasis }\end{aligned}$ & $\begin{array}{l}22 \\
15 \\
29 \\
27\end{array}$ & $\begin{array}{l}308000 \\
326900 \\
336500 \\
337100\end{array}$ & $\begin{array}{l}183900 \\
297600 \\
309700 \\
320000\end{array}$ & $\begin{array}{l}545500 \\
469600 \\
574200 \\
508700\end{array}$ & $\begin{array}{l}303700^{\prime} \\
338700 \\
517300 \\
493600\end{array}$ & $\begin{array}{l}1,77 \\
1,44 \\
1,71 \\
1,51\end{array}$ \\
\hline II & $\begin{array}{l}\text { Stühle in welchen die Fraktionen } 4 \mathrm{a} \text { und } 1 \\
\text { und (oder) } 1 \text { a gefunden waren. }\end{array}$ & 22 & 142800 & 193900 & 134100 & 149500 & 0,95 \\
\hline III & $\begin{array}{l}\text { Stühle in welchen nur die Fraktion } 4 \text { a } \\
\text { gefunden war. }\end{array}$ & 25 & 47800 & 32400 & 35100 & 24300 & 0,73 \\
\hline
\end{tabular}

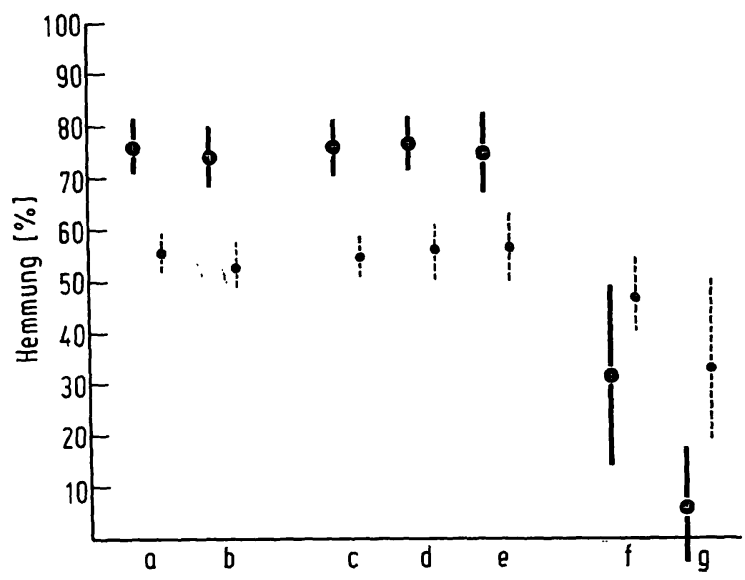

Abb. 3. Hemmung der alkalischen Phosphatase-Aktivität in Faecesextrakten durch $5 \mathrm{mmol} / \mathrm{l} L$-Phenylalanin. Gezeichnet sind Mittelwerte \pm Standardabweichungen der Herabsetzungen der Anfangsaktivität bei $\mathrm{pH} 9$ und pH 10 in Prozent. (pH $9-$ - pH $10---)$ ). a)-e) Stühle, in welchen die Fraktion 4 a nicht gefunden worden war:

a) von gesunden Kindern, b) von Kindern mit akuter Virushepatitis, c) von gesunden Erwachsenen, d) von Erwachsenen mit Lebererkrankungen, e) von Erwachsenen mit Lambliasis

f) Stühle, in welchen die Fraktion 4 a neben 1 und/oder 1 a gefunden wurde,

g) Stühle, in welchen nur die Fraktion 4 a gefunden wurde.

fast nicht beeinflußt und auch bei pH 10 nur wenig inaktiviert.

Denaturierungsversuche mit $2 \mathrm{~mol} / \mathrm{l}$ Harnstoff führten bei beiden pH-Werten in allen Proben zu Aktivitätsverlusten von $36-41 \%$.

\section{Gelfiltratione der Extrakte}

Durch Kolonnen von Sephadex G 100 wurden filtriert:

1. ein Extrakt aus Faeces eines gesunden Erwachsenen,

2. ein Extrakt aus acholischen Faeces eines Kranken mit Stauungsikterus,
3. ein Extrakt aus Faeces eines erwachsenen Lamblienträgers in welchem nur die Fraktion 4 a gefunden worden war,

4. ein Extrakt aus Dünndarmschleimhaut,

5. ein Extrakt aus Blasengalle.

Auf der Abbildung 4 sind die Elutionsdiagramme dargestellt. Aus den Kolonnen wurden zuerst inaktive Eiweißstoffe ausgewaschen. Die nachfolgenden Phosphatase-aktiven Portionen enthielten nur wenig bei $280 \mathrm{~nm}$ Licht absorbierendes Material (Abb. 4a, b). Kurve a der alkalischen Phosphatase (normale Faeces) weist auf zwei nahverwandte Fraktionen hin, welche den Elektrophoresebanden 1 und 1 a entsprechen könnten. Dasselbe bedeutet wahrscheinlich auch die linke Schulter auf Kurve b (acholische Faeces).

Die breite Kurve der Fraktion 4a auf Diagramm c spricht dafür, daß̧ das eluierte Enzym nicht homogen war. Im Einklang damit steht die stets breite Zone der. Fraktion 4a auf Elektropherogrammen.

Das Diagramm d zeigt, daß die alkalische Phosphatase der Dünndarmschleimhaut in Bezug auf Molekülgröße sehr homogen war, obgleich sich auf Elektropherogrammen zwei Banden mit nur wenig verschiedener Beweglichkeit zeigten. Diagramm e zeigt, daß die alkalische Phosphatase aus Blasengalle etwas früher eluiert wurde

Tab. 3. Vergleich der alkalischen Phosphatasenaktivitäten in Rohextrakten und Eluaten. King-Armstrong-Einheiten, pH 9.

\begin{tabular}{lccc}
\hline Phosphatasen & \multicolumn{2}{l}{$\begin{array}{l}\text { Phosphatasenaktivitäten } \\
\text { eingetragen }\end{array}$} & $\begin{array}{l}\text { eluiert } \\
\text { wieder- } \\
\text { gefunden (\%) }\end{array}$ \\
\hline $\begin{array}{l}\text { Faeces eines gesunden } \\
\text { Erwachsenen }\end{array}$ & 12,6 & 12,8 & 101 \\
Acholische Faeces & 40,0 & 35,2 & 88 \\
$\begin{array}{l}\text { Faeces mit der } \\
\text { Fraktion 4a }\end{array}$ & 6,0 & 1,9 & 31 \\
$\begin{array}{l}\text { Darmschleimhaut- } \\
\text { extrakt }\end{array}$ & 26,2 & 26,6 & 101 \\
Gallenextrakt & 1,6 & 5,6 & 350 \\
\hline
\end{tabular}



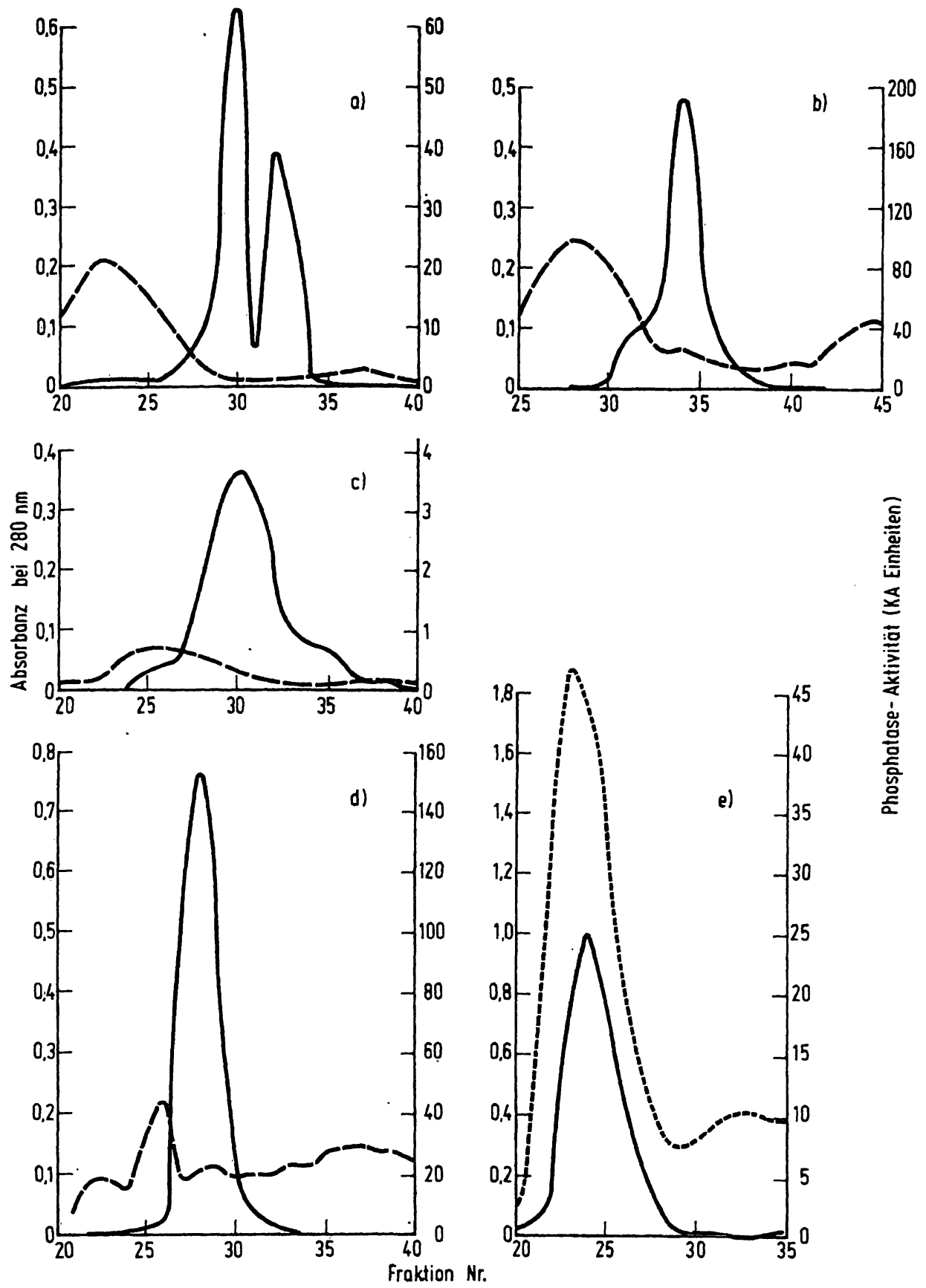

Abb. 4. Elutionsdiagramme der alkalischen Phosphatasen aus den Sephadex G 100 Säulen.

— Aktivität der alkalischen Phosphatasen, King-Armstrong-Einheiten

- - Protein, Absorbanz bei $280 \mathrm{~nm}$

... Gallenpigmente, Absorbanz bei $280 \mathrm{~nm}$.

a) Normale Faeces

b) Acholische Faeces

c) Faeces, die Fraktion 4 a enthalten

d) Darmschleimhaut

e) Galle

als die alkalische Phosphatase aus Faeces. Das Eluat war grünlich gefärbt und absorbierte stark im UV.

Auf der Tabelle 3 sind die Aktivitäten der alkalischen Phosphatase der gesammelten Eluate mit denen des Ausgangsmaterial's verglichen.

Die alkalische Phosphatase aus den Faeces einer gesunden Person und aus Darmschleimhaut wurden in den Eluaten vollständig wiedergefunden. Von der Aktivität der alkalischen Phosphatase im Extrakt aus acholischem Stuhl wurde ein kleiner Teil, von der aus Stuhl mit der
Fraktion 4a mehr als zwei Drittel im Gelfiltrat nicht wiedergefunden.

Das Elutionsdiagramm c auf der Abbildung 4 entspricht also nur etwa einem Drittel der in die Kolonne eingetragenen alkalischen Phosphatase. Dagegen zeichnete sich der Gallenextrakt dadurch aus, daß die Aktivität des Eluats mehr als verdreifacht war. Wir vermuten, daß in der Kolonne Gallensäuren zurückgeblieben waren, welche nach Peterlik (11) alkalische Phosphatase stark inhibieren. 
Untersuchungen der durch Gelfiltration gereinig: ten alkalischen Phosphatase

Die eluierten alkalischen Phosphatasen wurden nochmals mittels Stärkegelelektrophorese aufgetrennt, mit $L$ Phenylalanin inkubiert, bei $56^{\circ} \mathrm{C}$ inaktiviert, sowie ihre spezifischen Aktivitäten und $\mathrm{K}_{\mathrm{m}}$-Werte bestimmt.

Nach der Filtration über Sephadex konnten wir verschiedene schwache, in der Nähe der Auftragsstelle zurückbleibende Fraktionen auf Elektropherogrammen nicht wiederfinden. Die Lage der Hauptfraktionen aus normalem Stuhl und Dünndarmschleimhaut war unverändert. Im gereinigten Extrakt aus acholischem Stuhl fehlte die Fraktion 3. Die Hauptfraktion der alkalischen Phosphatase aus Galle erschien bei gleicher, geringer Beweglichkeit kompakter als vor der Reinigung. Die Fraktion 4a war im Elektropherogramm des nur wenig aktiven Eluats nicht sichtbar.
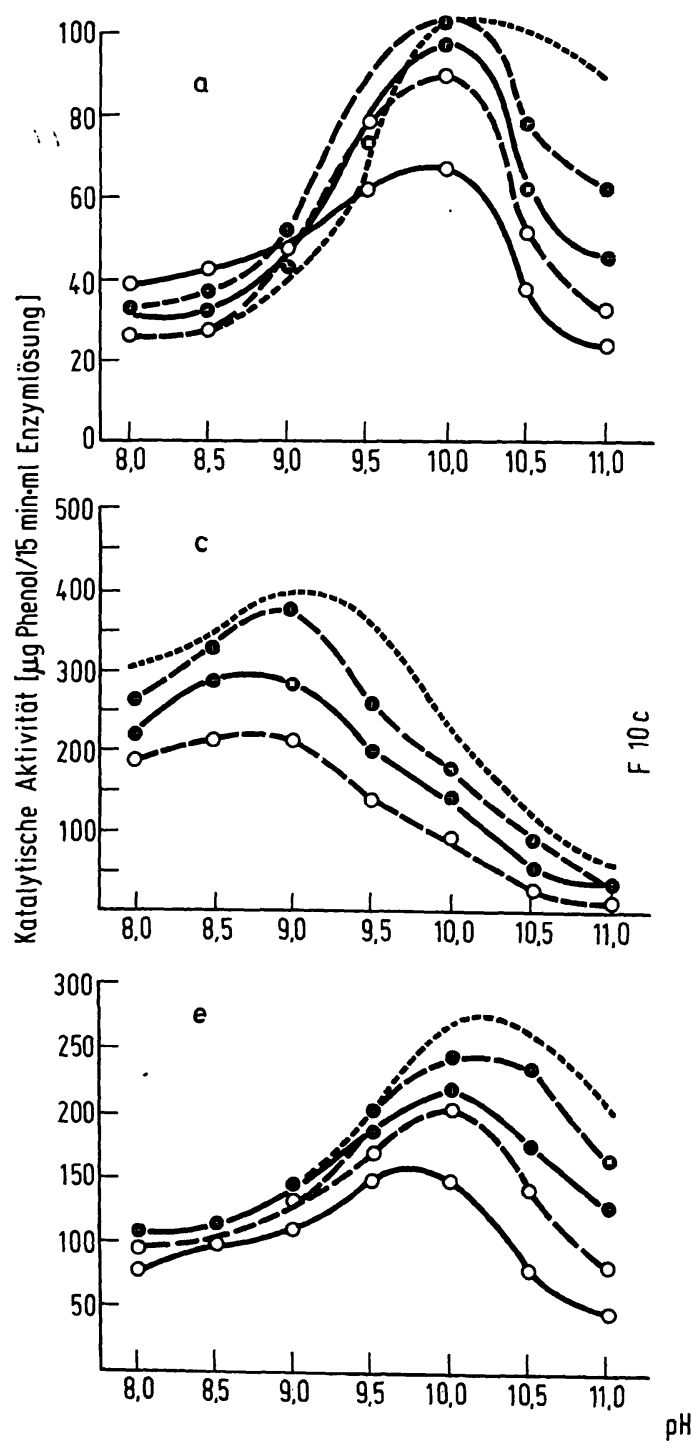

Abb. 5. pH-Optima der gereinigten alkalischen Phosphatasen bei verschiedenen Konzentrationen von Phenylphosphat:

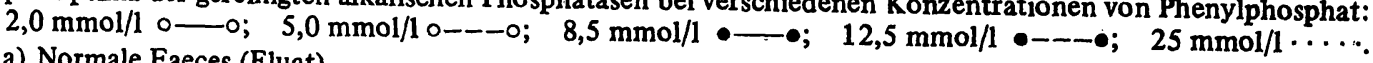

a) Normale Faeces (Eluat)

b) Acholische Faeces (Eluat)

c) Faeces, die Fraktion 4 a enthalten (Extrakt)
Alkalische Phosphatasen aus normalem und acholischem Stuhl, aus Dünndarmschleimhaut und auch aus Galle wurden vor und nach der Gelfiltration durch $L$-Phenylalanin sowohl bei $\mathrm{pH} 9$ als auch bei $\mathrm{pH} 10$ fast ebenso stark inhibiert wie vor der Reinigung, zugleich waren sie nach der Reinigung viel empfindlicher gegen erhöhte Temperatur als in rohen Extrakten. Mit der gereinigten. Fraktion 4a konnten wir keine Inhibitions- und Inaktivierungsversuche ausführen, weil ihre Aktivität im Eluat zu gering war.

Resultate der Aktivitätsmessungen bei verschiedenem $\mathrm{pH}$ mit verschiedenen Substratkonzentrationen sind auf Abbildung 5 dargestellt.

Die pH Optima der alkalischen Phosphatase aus normalem und acholischem Stuhl, Dünndarmschleimhaut und Galle liegen alle bei $\mathrm{pH} \mathrm{10,} \mathrm{das} \mathrm{Optimum} \mathrm{der}$ Fraktion 4 a liegt dagegen bei $\mathrm{pH} 9$.
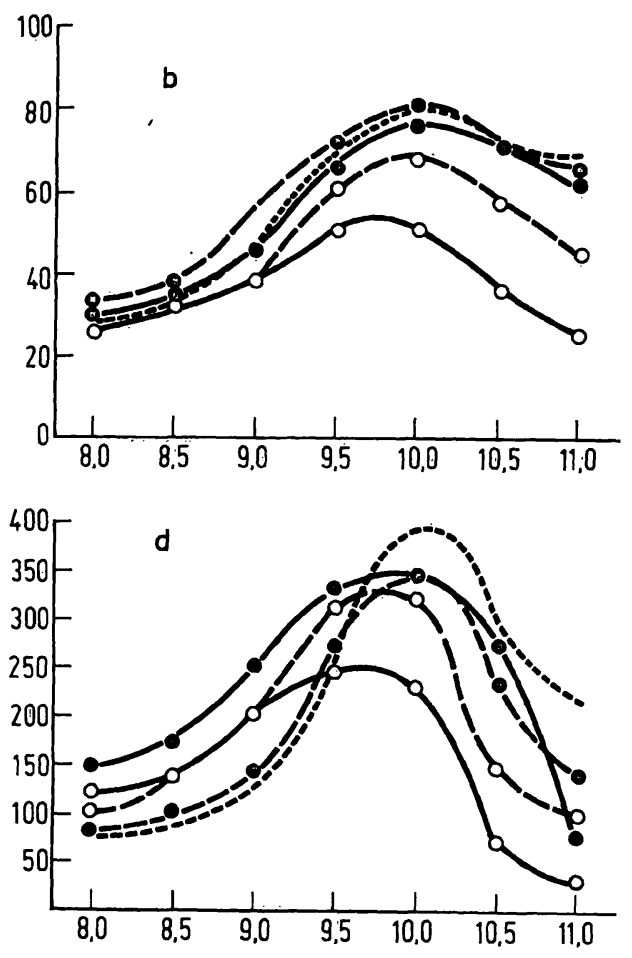

(Eluat)

e) Galle (Eluat) 
Tab. 4. $\mathbf{K}_{\mathbf{m}}$-Werte der alkalischen Phosphatasen aus Stühlen, Dünndarmschleimhaut und Galle. Substrat: Dinatriummonophenylphosphat.

\begin{tabular}{lcc}
\hline Phosphatasen & $\begin{array}{c}\mathrm{V}_{\max } \\
(\mu \mathrm{g} / \mathrm{ml} \text { Phenol })\end{array}$ & $\begin{array}{l}\mathrm{K}_{\mathrm{m}} \\
(\mathrm{mmol} / 1 \\
\text { Phenylphosphat })\end{array}$ \\
aus & & \\
\hline Faeces eines gesunden & 120,4 & 1,52 \\
Erwachsenen & 91,0 & 1,52 \\
Acholische Faeces & 16,6 & 10,0 \\
Faeces mit der Fraktion 4a & 416,6 & 1,52 \\
Darmschleimhautextrakt & 294,1 & 2,04 \\
Gallenextrakt & \\
\hline
\end{tabular}

$\mathrm{K}_{\mathrm{m}}$-Werte sind in der Tabelle 4 angeführt.

Alkalische Phosphatasen aus normalem, acholischem Stuhl und Dünndarmschleimhaut hatten identische, niedrige $\mathrm{K}_{\mathrm{m}}$-Werte, Gallenphosphatase einen nur wenig höheren. Mehr als sechsfach höher war der $K_{m}$-Wert der Fraktion $4 \mathrm{a}$.

\section{Untersuchung der Fraktion 4 a}

Alle beschriebenen Beobachtungen wiesen darauf hin, daß die Fraktion 4a synthetische Phosphorsäureester vom Typus Phenyl- oder Naphthylphosphat zwar spaltete, aber eine viel geringere Affinität für solche Ester hatte als die typischen alkalischen Phosphatasen. Der Aktivitätsverlust bei Gelfiltration ließ uns vermuten, daß es sich bei dieser Fraktion um eine Phosphatase mit größerem Molekülgewicht handeln könnte. Als Kandidat kam die zuerst von Reis (12) in vielen menschlichen Geweben gefundene $5^{\prime}$-Nucleotidase in Betracht, welche nach Kowlessar $(13,14)$ und Taswell $(15)$ in Stärkegelelektropherogrammen im Bereich zwischen Auftragstelle und $\alpha_{2}$ Globulinen zurïckbleibt, wo wir die Fraktion 4a auch gefunden haben. Moss (16) hat 1966 versucht, die 5'-Nucleotidase von den unspezifischen alkalischen Phosphatasen zu trennen, wobei er fand, daß die 5'-Nucleotidase ein Molekulargewicht von mehr als 200.000 haben müsse und daß sie nur geringe Affinität für Phenylphosphat hat.

Deshalb bestimmten wir die Aktivitäten der alkalischen Phosphatasen und der 5'-Nucleotidase nach Campbell. Die Resultate dieser Versuche sind in der Tabelle 5 angegeben.

Tab. 5. Anteil der 5'-Nucleotidase (in Prozenten) an der gesamten alkalischen Phosphatasenaktivität von Stühlen, Dünndarmschleimhaut und Galle. Messungen nach Campbell (10).

\begin{tabular}{|c|c|c|c|}
\hline Phosphatasen & $\begin{array}{l}\text { Gesamt- } \\
\text { aktivität }\end{array}$ & $\begin{array}{l}\text { Aktivit } \\
\text { cleotida } \\
\text { mg P/1 }\end{array}$ & $\begin{array}{l}\text { der 5'-Nu- } \\
\text { \% der } \\
\text { Gesamtak } \\
\text { tivität }\end{array}$ \\
\hline $\begin{array}{l}\text { Faeces eines gesunden } \\
\text { Erwachsenen } \\
\text { Acholische Faeces } \\
\text { Faeces mit der Fraktion 4a } \\
\text { Darmschleimhautextrakt } \\
\text { Gallenextrakt }\end{array}$ & $\begin{array}{l}25.8 \\
21.7 \\
10.0 \\
13.6 \\
11.4\end{array}$ & $\begin{array}{l}4.8 \\
5.1 \\
4.9 \\
3.3 \\
1.4\end{array}$ & $\begin{array}{l}18,6 \\
23,8 \\
49,0 \\
24,3 \\
12,2\end{array}$ \\
\hline
\end{tabular}

In jedem der untersuchten Extrakte war ein Teil, höchstens ein Viertel, der alkalischen PhosphataseAktivität auch mit Adenosin-5-phosphat bei pH 7,5 meßbar. Eine Ausnahme bildete wieder der Extrakt aus Stuhl mit der Fraktion $4 a$, in welchem die Hälfte der Gesamtaktivität von 5'-Nucleotidase zu stammen schien.

\section{Diskussion}

Der Ausgangspunkt unserer Untersuchungen der alkalischen Phosphatasen der Faeces waren die Fragen, woher diese Enzyme stammten, und, ob es bei Störungen im Bereich der Verdauungsorgane zu charakteristischen Änderungen ihrer Ausscheidung käme.

Unsere Beobachtungen sprechen dafür, daß der Hauptteil der alkalischen Phosphatase in normalen und acholischen Faeces aus der Dünndarmschleimhaut stammt, und daß normalerweise nicht viel Gallenphosphatase im Stuhl vorkommt. Die alkalische Phosphatase der normalen und acholischen Faeces hat das gleiche $\mathrm{pH}-\mathrm{Optimum}$ und den gleichen $\mathrm{K}_{\mathrm{m}}$-Wert wie die Dünndarmphosphatase, wird durch $L$-Phenylalanin gleich inhibiert und beim Erwärmen in gleichem Maß inaktiviert. Es scheint aber, daß die alkalische Phosphatase der Schleimhaut im Darm nicht unverändert bleibt, da sich der Hauptteil der alkalischen Phosphatase in Faeces von der in Schleimhaut durch etwas größere Beweglichkeit bei Stärkegelelektrophorese unterscheidet.

Alkalische Phosphatase aus Galle hat einen höheren $\mathrm{K}_{\mathbf{m}}$-Wert, viel geringere Beweglichkeit im Elektropherogramm, auch wird sie durch $L$-Phenylalanin - im Gegensatz zu den alkalischen Phosphatase aus Stühlen und Schleimhaut - kaum gehemmt.

Unsere zweite Frage glauben wir bejahend beantworten zu können. Die von uns „4 a“" genannte Faeces-Phosphatase, welche Eigenschaften einer $5^{\prime}$-Nucleotidase hat, haben wir in Stühlen von Kranken mit akuter Virushepatitis, Lebercirrhose und Protozoasis beobachtet. Es könnte sein, daß ihr Auftreten mit Störungen verbunden ist, welche tiefere Gewebsschichten erfassen. Protozoen vermehren sich in der Schleimhaut des Darms und der Gallenwege und zerstören die oberflächlichen Zellschichten; die 5'-Nucleotidase der Gewebe, welche nicht wasserlöslich ist, könnte dann unter dem Einfluß der Gallensäuren herausgelöst und mit der Galle in den Darm gebracht werden.

Bemerkenswert scheint uns, daß das Auftreten der Fraktion 4a im Stuhl stets von einem starken Abfall der gesamten Aktivität begleitet ist. Es sieht so aus, als ob die Produktion der alkalischen Phosphatase in der Schleimhaut, oder ihre Ausscheidung in den Darm, gehemmt sei.

Die Feststellung der beschriebenen Veränderungen des Gehalts von alkalischer Phosphatase im Stuhl könnte einen Beitrag zur Diagnostik der Leber- und Darmkrank- 
heiten bilden. Es ist nicht nötig, die Fraktion 4 a mittels Elektrophorese aufzusuchen. Bestimmungen der Phos-

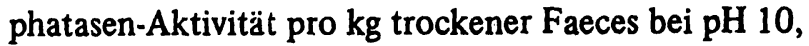

und der Inhibition durch $L$-Phenylalanin bei $\mathrm{pH} 9$ und 10 genügen zur Charakterisierung der abnormalen Enzymlage im Darm.

\section{Literatur}

1. Benić, V. \& FiY̌er-Herman, M. (1970), diese Z. 8, 458-464.

2. Kirberger, E. \& Martini, G. A. (1950), Deut. Arch. Klin. Med. 197, 268-287.

3. King, E. J. \& Armstrong, A. R. (1934), Can. Med. Ass. J. 31 , $376-381$.

4. Fishman, W. H. \& Ghosh, N. K. (1967), Advan. Clin. Chem. $10,225-270$.

5. Fennelly, J. J., Fitzgerald, M. X. \& Geeney, K. Mc. (1969), Gut 10, 45-51.

6. Posen, S., Neale, F. C. \& Clubb, J. S. (1965), Ann. Intern Med. 62, 1234-1243.

7. Smithies, O. (1955), Biochem. J. 61, 629-641.

8. Poulik, M. D. (1957), Nature (London) 180, 1477-1479.

9. Bamford, K. F., Harris, H., Luffman, J. E., Robson, E. B. \& Cleghorn, T. E. (1965), Lancet I, 530-531.

10. Campbell, D. M. (1962), Biochem. J. 82, 34 P.

11. Peterlik, M. (1966), diese Z. 4, 34-35.

12. Reis, J. L. (1951), Biochem. J. 48, 548-551.

13. Kowlessar, O. D., Pert, J. H., Haeffner, L. J. \& Sleisenger, M. H. (1959), Proc. Soc. Exp. Biol. Med. 100, 191-193.

14. Kowlessar, O. D., Haeffner, L. J. \& Riley, E. M. (1961), Ann. N. Y. Acad. Sci. 94, 836-843.

15. Taswell, H. F. \& Jeffers, D. M. (1963), Amer. J. Clin. Pathol. 40, 349-356.

16. Moss, D. W. (1966), Nature (London) 209, 806-807.

Dr. mr. Vera Benić

Klinika za zarazne bolesti 41000 Zagreb, Mirogojska 8 Jugoslavija

Prof. dr. Marijana Fišer-Herman 41000 Zagreb, Bijenička 21 Jugoslavija 